\title{
Overcrowding Effects on Larval Development of Four Mosquito species Aedes albopictus, Aedes aegypti, Culex quinquefasciatus and Anopheles stephensi
}

\author{
Ruchi Yadav ${ }^{1}$, Varun Tyagi ${ }^{2}$, Ajay K Sharma ${ }^{1}$, Sachin N Tikar ${ }^{1}$, Devanathan Sukumaran ${ }^{1}$, \\ Vijay Veer ${ }^{2}$ \\ ${ }^{1}$ Vector Management Division, Defence Research and Development Establishment (DRDE), Jhansi Road, \\ Gwalior M. P., India \\ ${ }^{2}$ Defence Research Laboratory, Tezpur, Assam, India
}

*Corresponding Author: Varun Tyagi, Defence Research Laboratory, Tezpur, Assam, India

\begin{abstract}
Mosquitoes are recognized as medically important vectors, responsible for various disease transmissions. These mosquito species are vastly abundant in artificial containers and strongly adapted to urban environment. Forest clearing, irrigation development and human resettlement results in large-scale increase of artificial containers, and provides various types of habitat for mosquito breeding. To understand how intra-specific competition affects larval growth we studied survivorship, development time and adult dry weight of four mosquito species Ae, albopictus, Ae. aegypti, Cx. quinquefasciatus and An. stephensi provided controlled food and space, under laboratory conditions. Larvae were reared at 5 different densities - 20, 50, 100, 150 and 200 larvae / $200 \mathrm{ml}$ water with constant water volume; food amount and under controlled temperature and photoperiod. Larval survivorship, development time and adult dry weight were observed as the parameters of larval development for each density and data were subjected to one-way ANOVA. The analysis showed that larval density was strongly associated with increased development time and decreased survival and dry weight of adult mosquitoes. Larval growth of Cx quinquefasciatus and An. stephensi larvae especially those from higher density, was significantly affected as compared to Aedes larvae. It was also observed that Cx quinquefasciatus and An. stephensi both are comparatively sensitive to crowding in small containers. Male-biased sex proportion at higher densities was also observed for each mosquito species. The findings are important in establishing the relationship between models of mosquito population regulation and epidemiological models of disease transmission.
\end{abstract}

Keywords: larval competition, Aedes albopictus, Aedes aegypti, Culex quinquefasciatus, Anopheles stephensi, overcrowding, larval growth.

\section{INTRODUCTION}

Aedes albopictus, Aedes aegypti, Anopheles stephensi and Culex quinquefasciatus mosquitoes are recognized as medically important vectors, responsible for various disease transmissions such as Dengue, Chikungunya, malaria and Japanese encephalitis. These mosquito species are vastly abundant in artificial containers and strongly adapted to urban environment. Forest clearing, irrigation development and human resettlement results in large-scale increase of artificial containers, and provides various types of habitat for mosquito breeding ${ }^{(1,2)}$. Aigbodion and Uyi $(2013)^{(3)}$ have shown a high frequency of $C x$. quinquefasciatus species, either alone or together in certain waters and sometimes even together with species of An. stephensi and Aedes species. Anopheline species are known to be ground pool breeders, although large numbers have been observed in gutters, periodomestic runoff and domestic containers ${ }^{(4,5)}$. As a result of adaptation to human environments, mosquito larvae may develop in man-made containers which are able to hold stagnant water. Resource competition in such type of small water containers could undergo intra-specific or interspecific competition. This competition promotes density-dependent interactions among individuals which lead to reduced mosquito survivorship, growth, and adult size ${ }^{(6,7)}$. Gimnig et al. (2002) ${ }^{(8)}$ observed the significant differences in size and larval survivorship, while studying the densitydependent larval interactions in An. gambiae. 
Mosquito population in a particular habitat is determined by extrinsic factors included weather, climate and geographical features and intrinsic factors such as food and space ${ }^{(9)}$. Intrinsic or density dependent factors are very important elements, in models of diseases transmission ${ }^{(10)}$. Effects of competitive interactions among larval stages may induce differences in adult life traits such as variation in size may alter vector-virus interactions. Reproduction of mosquito could be affected due to the deficiency of nutrition at larval stages ${ }^{(11)}$ and may alter disease transmission as well ${ }^{(12)}$. To understand how intra-specific competition affects larval growth we studied survivorship, development time and adult dry weight of four mosquito species Ae, albopictus, Ae. aegypti, Cx. quinquefasciatus and An. stephensi provided controlled food and space, under laboratory conditions. Based upon the present study we predicted that as a consequence of higher competition, adult population would negatively affected by taking long time to reach adulthood while reduced survival and adult fitness.

\section{Materials AND Methods}

Mosquitoes: All four species of mosquitoes were obtained from laboratory colony, originated from wild mosquitoes, collected from Gwalior MP India. Mosquito colonies were maintained separately at $27 \pm 2^{\circ} \mathrm{C}$ room temperature and $70 \pm 10 \%$ relative humidity in our laboratory in DRDE, Gwalior, India. Larval stages were maintained in bowls $(2.5 \mathrm{~L})$ by providing yeast powder as larval food. Adult mosquitoes were reared in wooden cages (30x30x30 inches) and were provided cotton soaked with $10 \%$ sugar solution. Ae. albopictus, Ae. aegypti and An. stephensi females were offered rabbit while $C x$. quinquefasciatus were offered fowl once a week for blood feeding. Water filled dishes lined with filter paper were kept inside the cage for egg laying. Eggs of each mosquito species were collected and washed with dechlorinated water. Eggs were air-dried for $2 \mathrm{hrs}$ and then transferred to water filled bowls for hatching. Newly hatched ( 24 hrs old) F2 larvae were used for experiment.

Experimental Design: Five different larval densities of 20, 50, 100, 150 and 200 larvae/ $200 \mathrm{ml}$ water were studied for the overcrowding effect on larval development. Three replications for each larval density were set up in plastic bowls (sized $500 \mathrm{ml}$ volume, $12 \mathrm{~cm}$ diameter and $6 \mathrm{~cm}$ depth) filled with $200 \mathrm{ml}$ dechlorinated water. Newly hatched larvae were counted according to selected densities and transferred to experimental bowls. Water level was maintained throughout the study and $15 \mathrm{mg}$ of yeast powder was added to the bowls daily until all the larvae were pupated. This amount of food was considered in excess for uncrowded densities ( $20 \& 50$ larvae/ $200 \mathrm{ml}$ water), while at more crowed densities ( 150 \& 200 larvae/ $200 \mathrm{ml}$ water) it was limited according to the optimum food amounts suggested by Suleman $(1982){ }^{(13)}$. The whole experiment was performed at laboratory conditions mentioned earlier and observations were started after $24 \mathrm{hrs}$. Emerged adult mosquitoes were counted and separated and the dead larvae were also removed and counted daily. Adult mosquitoes were killed and kept for freeze drying. After $48 \mathrm{hrs}$ dried adults were weighed and all the observations were recorded until the last larvae pupated and emerged out as adult from each replicates.

Statistical Analysis: Three parameters- survivorship, development time and adult dry weight were studied to evaluate the effects of larval density on larval growth. Survivorship was estimated as the percentage of larvae attaining metamorphosis and reaching adulthood. Development time was calculated from the beginning of egg stage to the day of last adult emergence for each container. Adult dry weight was measured, for all individuals reaching the adult stage in each treatment. To examine the importance of all variables on larval growth of different species, all outcomes were subjected to One Way ANOVA ${ }^{(14)}$. The relationship between larval density and attributes of physical fitness was established using linear regression analysis. All the statistics were analyzed using Sigma Stat Statistical software.

\section{RESUltS}

The outcomes of one way ANOVA demonstrated the significant effect of larval crowding on mosquito development (Table.1). Results of larval crowding have shown variation in fitness parameters (survivorship, development time and adult dry weight) with species.

Survival: For all treatments, survival decreased with increasing larval density (Fig 1). At lowest larval density (20 larvae/ $200 \mathrm{ml}$ water) all four mosquito species showed high survival rate $(80-90$ $\%)$. According to statistical outcomes, it was observed that from lower (20 larvae /container) to medium larval density (50 larvae / $200 \mathrm{ml}$ water), the survivorship of individual was $>80 \%$ while at 
Overcrowding Effects on Larval Development of Four Mosquito species Aedes albopictus, Aedes aegypti, Culex quinquefasciatus and Anopheles stephensi

higher densities (150 and 200 larvae / 200 ml water) it fall between 40-55 \%. Both the Aedes species showed almost similar pattern of highest survival as compared to Anopheles and Culex. The survival rate of An. stephensi was strongly affected with larval density (Survival $=2.45-0.349 \log$ density; $P<$ $0.001 ; \mathrm{R}^{2}=0.872$ ) among all the four species. In Culex quinquefasciatus the survivorship was 83 and $84 \%$ at lower and medium larval density respectively, it turned down drastically at higher densities.

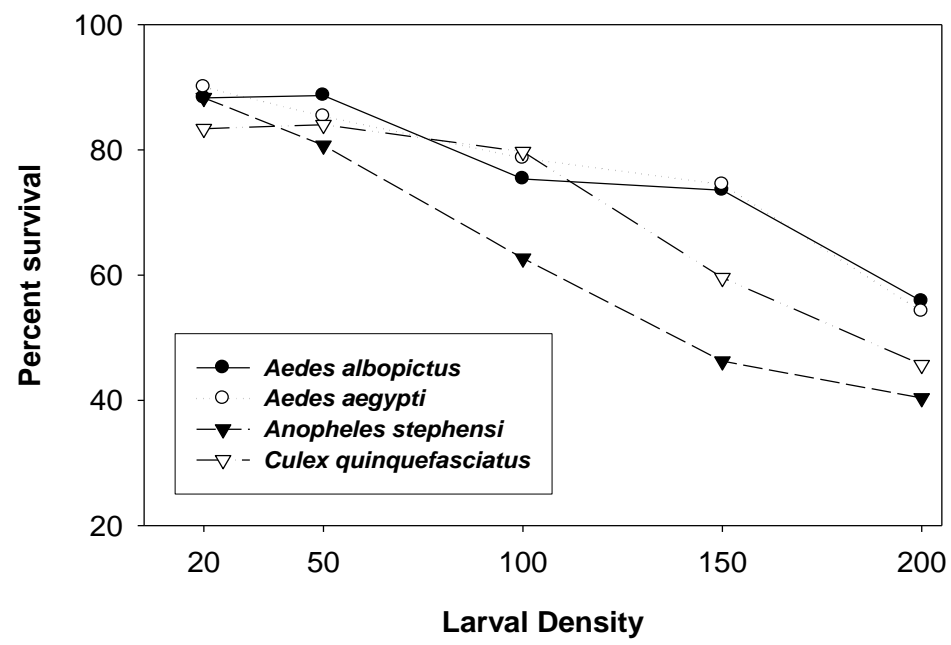

Figure 1. Relationship between Larval density and survivorship

Table 1. One Way ANOVA for Survivorship, Development time and Adult dry weight of different mosquito species raised at different larval density.

\begin{tabular}{|c|c|c|c|c|}
\hline Response & & MS & $\mathbf{F}$ & $P$ \\
\hline \multicolumn{5}{|l|}{ Ae. albopictus } \\
\hline \multirow[t]{2}{*}{ Survivorship* } & $\mathrm{M}$ & 1370.933 & 158.185 & $<0.001$ \\
\hline & $\mathrm{F}$ & 1173.067 & 96.681 & $<0.001$ \\
\hline \multirow[t]{2}{*}{ Adult dry weight } & $\mathrm{M}$ & 0.0374 & 15.770 & $<0.001$ \\
\hline & $\mathrm{F}$ & 0.197 & 6.943 & 0.006 \\
\hline Development Time** & Pooled & 36.433 & 26.024 & $<0.001$ \\
\hline \multicolumn{5}{|l|}{ Ae. aegypti } \\
\hline \multirow[t]{2}{*}{ Survivorship } & $\mathrm{M}$ & 1418.733 & 55.419 & $<0.001$ \\
\hline & $\mathrm{F}$ & 1111.267 & 200.831 & $<0.001$ \\
\hline \multirow[t]{2}{*}{ Adult dry weight } & $\mathrm{M}$ & 0.0590 & 25.428 & $<0.001$ \\
\hline & $\mathrm{F}$ & 0.493 & 31.926 & $<0.001$ \\
\hline Development Time & Pooled & 22.233 & 7.940 & 0.004 \\
\hline \multicolumn{5}{|l|}{ An. stephensi } \\
\hline \multirow[t]{2}{*}{ Survivorship } & $\mathrm{M}$ & 631.833 & 81.004 & $<0.001$ \\
\hline & $\mathrm{F}$ & 349.933 & 82.016 & $<0.001$ \\
\hline \multirow[t]{2}{*}{ Adult dry weight } & $\mathrm{M}$ & 0.0455 & 64.986 & $<0.001$ \\
\hline & $\mathrm{F}$ & 0.302 & 18.626 & $<0.001$ \\
\hline Development Time & Pooled & 14.500 & 6.797 & 0.007 \\
\hline \multicolumn{5}{|l|}{ Cx. quinquefasciatus } \\
\hline \multirow[t]{2}{*}{ Survivorship } & $\mathrm{M}$ & 971.233 & 57.583 & $<0.001$ \\
\hline & $\mathrm{F}$ & 683.100 & 43.234 & $<0.001$ \\
\hline \multirow[t]{2}{*}{ Adult dry weight } & $\mathrm{M}$ & 0.0385 & 32.438 & $<0.001$ \\
\hline & $\mathrm{F}$ & 0.528 & 36.866 & $<0.001$ \\
\hline Development Time & Pooled & 45.933 & 19.139 & $<0.001$ \\
\hline
\end{tabular}

Survivorship*- Total number of emerged adults

Development Time ${ }^{* *}$ - From the egg to Emergence of last larvae

Analysis showed that larval density also affects the male and female ratio of mosquitoes (Figure 2). More males reached the adult stage at higher larval densities, while maximum females were emerged 
Overcrowding Effects on Larval Development of Four Mosquito species Aedes albopictus, Aedes aegypti, Culex quinquefasciatus and Anopheles stephensi

at lower densities. All the four species showed deviation from 1:1 sex ratio in higher densities (Table 2). It was also observed that strength of deviation was lowest in Ae. albopictus as compared to Ae. aegypti, Cx. quinquefasciatus and An. stephensi species.

A

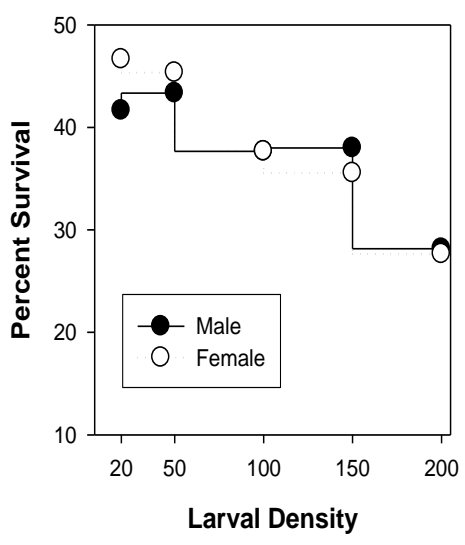

B

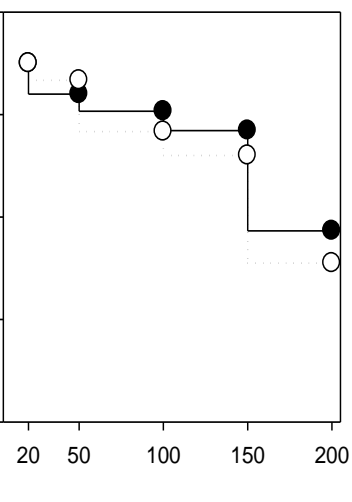

C

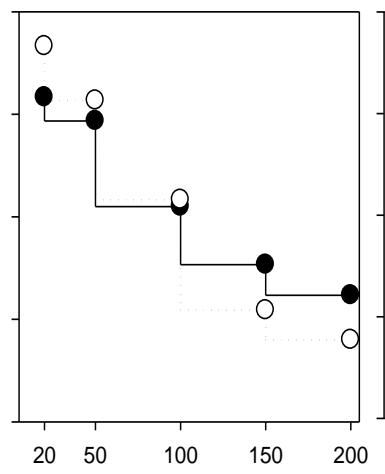

D

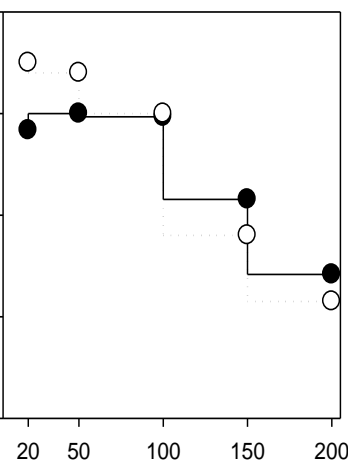

Figure 2. Effect of Larval density on survivorship of different mosquito species

(A-Aedes. albopictus; B -Aedes. aegypti; C-Anopheles stephensi; D-Culex quinquefasciatus)

Table 2. Male and Female ratio of total emerged mosquitoes at different larval densities (Larvae /200 ml)

\begin{tabular}{|c|c|c|c|c|c|}
\hline Mosquito Species & Larval Density & $\mathbf{N}$ & Male :Female & $\chi^{2}$ & $\boldsymbol{P}$ \\
\hline Ae. albopictus & & & & 0.559 & 0.967 \\
\hline & 20 & 60 & $25: 28$ & & \\
\hline & 50 & 150 & $65: 68$ & & \\
\hline & 100 & 300 & $113: 112$ & & \\
\hline & 150 & 450 & $171: 160$ & & \\
\hline Ae. aegypti & 200 & 600 & $169: 166$ & & \\
\hline & 20 & 60 & $27: 27$ & & 0.965 \\
\hline & 50 & 150 & $63: 65$ & & \\
\hline & 100 & 300 & $121: 115$ & & \\
\hline & 150 & 450 & $173: 162$ & & \\
\hline Cx. quinquefasciatus & 200 & 600 & $172: 153$ & & \\
\hline & 20 & & & 3.211 & 0.523 \\
\hline & 50 & 150 & $59: 28$ & & \\
\hline & 100 & 300 & $93: 95$ & & \\
\hline & 150 & 450 & $114: 94$ & & \\
\hline & 200 & 600 & $134: 108$ & & \\
\hline An. stephensi & & & & 2.040 & 0.728 \\
\hline & 20 & 60 & $23: 27$ & & \\
\hline & 50 & 150 & $60: 66$ & & \\
\hline & 100 & 300 & $119: 120$ & & \\
\hline & 150 & 450 & $142: 126$ & & \\
\hline & 200 & 600 & $145: 129$ & & \\
\hline
\end{tabular}

Development Time: According to Fig 3(A-D) all the four species exhibited similar tendency of development time where development time decreases with increase in larval density up to medium level, while at highest density it slightly increases. An. stephensi and $C x$. quinquefasciatus took longer durations (around 13-23 days and 14-19 days respectively) to attain adult stage as compared to Ae. albopictus (around 11-17 days) and Ae. aegypti (13-20 days) at all densities. An. stephensi and Cx. quinquefasciatus both took longest duration of (Mean) $23 \pm 0.577$ days and $19.33 \pm 1.333$ days respectively, at the larval density of 150 larvae/ Container. Larval density was found to be significantly associated with longer development time. This relationship was demonstrated by regression analysis - No. of days $=13.179+0.0265$ density, $R^{2}=0.384 ; 12.992+0.0430$ density, $R^{2}=$ 
Overcrowding Effects on Larval Development of Four Mosquito species Aedes albopictus, Aedes aegypti, Culex quinquefasciatus and Anopheles stephensi

$0.741 ; 13.561+0.0440$ density, $\mathrm{R}^{2}=0.595 ; 14.976+0.0227$ density, $\mathrm{R}^{2}=0.414$ at $P=<0.001$ for $A e$. albopictus, Ae. aegypti, An. stephensi and Cx. quinquefasciatus respectively.

Aedes albopictus
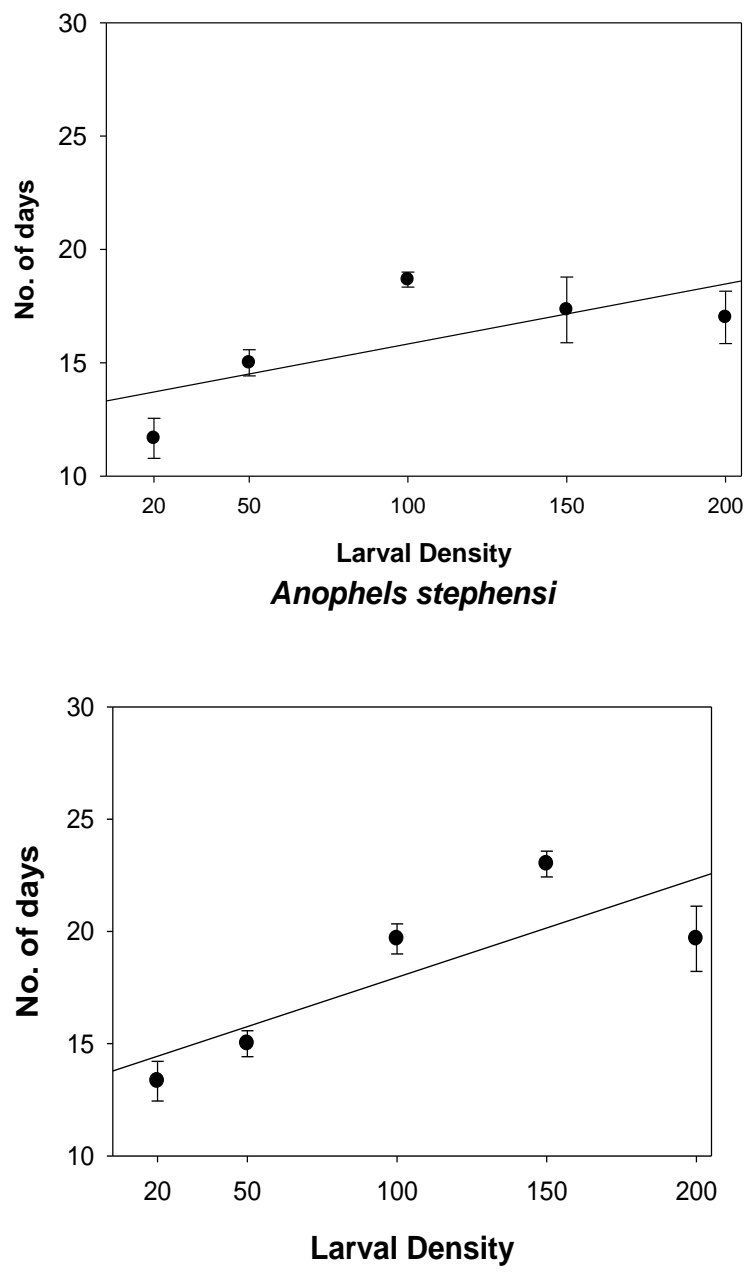

Aedes aegypti
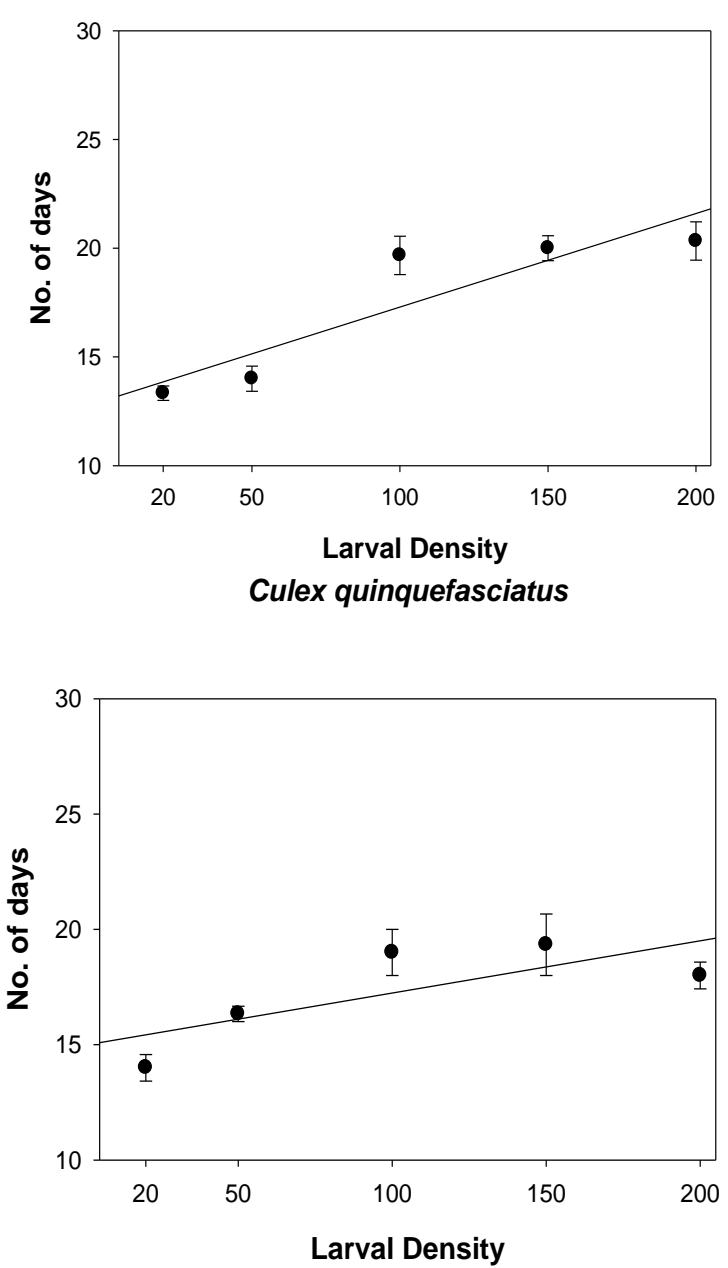

Figure 3. Association between Larval density and development time of different mosquito species.

Adult dry weight: Density effect was significant for both males and females, showing significant variation of adult dry weight among densities (Table 1). Density effect was almost similar for all the species where adult dry weight gradually decreased with population up to higher larval density while it slightly increased at highest Population (Figure 4). Dry weight of male Ae. albopictus ranged from 0.38-0.64 mg (mean) while female ranged from 0.61-1.13 mg at different larval densities. For Ae. aegypti it ranged from $0.36-0.68 \mathrm{mg}$ for males and $0.66-1.55 \mathrm{mg}$ for females. Among all species An. stephensi and $C x$. quinquefasciatus larvae were strongly affected with the larval density where larval competition hinders the adult development by reducing the weight $(\mathrm{F}=64.99,18.63$ for male and female An. stephensi; $32.44,36.87$ for male and female $C x$. quinquefasciatus respectively).

\section{DISCUSSION}

Present study suggests that apart from other environmental and climatic conditions such as temperature, humidity and competition with other species, larval crowding also influences the fitness of individuals and adaptation to space and food in mosquito life cycle. The variables studied in the present study represent the status of larval growth (survivorship, development time and adult dry weight) of different mosquito species. Our study clearly indicated that a higher larval density reduces the survivorship and weight of individuals and favored the longer development time. As a consequence of larval crowding, there is depletion of nutrients and release of various toxic wastes by individuals in the surrounding to overcome intraspecific competition ${ }^{(15,16,17)}$, which favors the larval 
Overcrowding Effects on Larval Development of Four Mosquito species Aedes albopictus, Aedes aegypti, Culex quinquefasciatus and Anopheles stephensi

mortality and hamper adult physical development ${ }^{(18,19)}$. It seems that turbidity in the water surface due to larval waste and bacterial growth may adversely affect mosquito larvae by reducing oxygen diffusion on the water surface or consuming dissolved oxygen in the water, and by mechanically hindering the siphonal respiration ${ }^{(20)}$
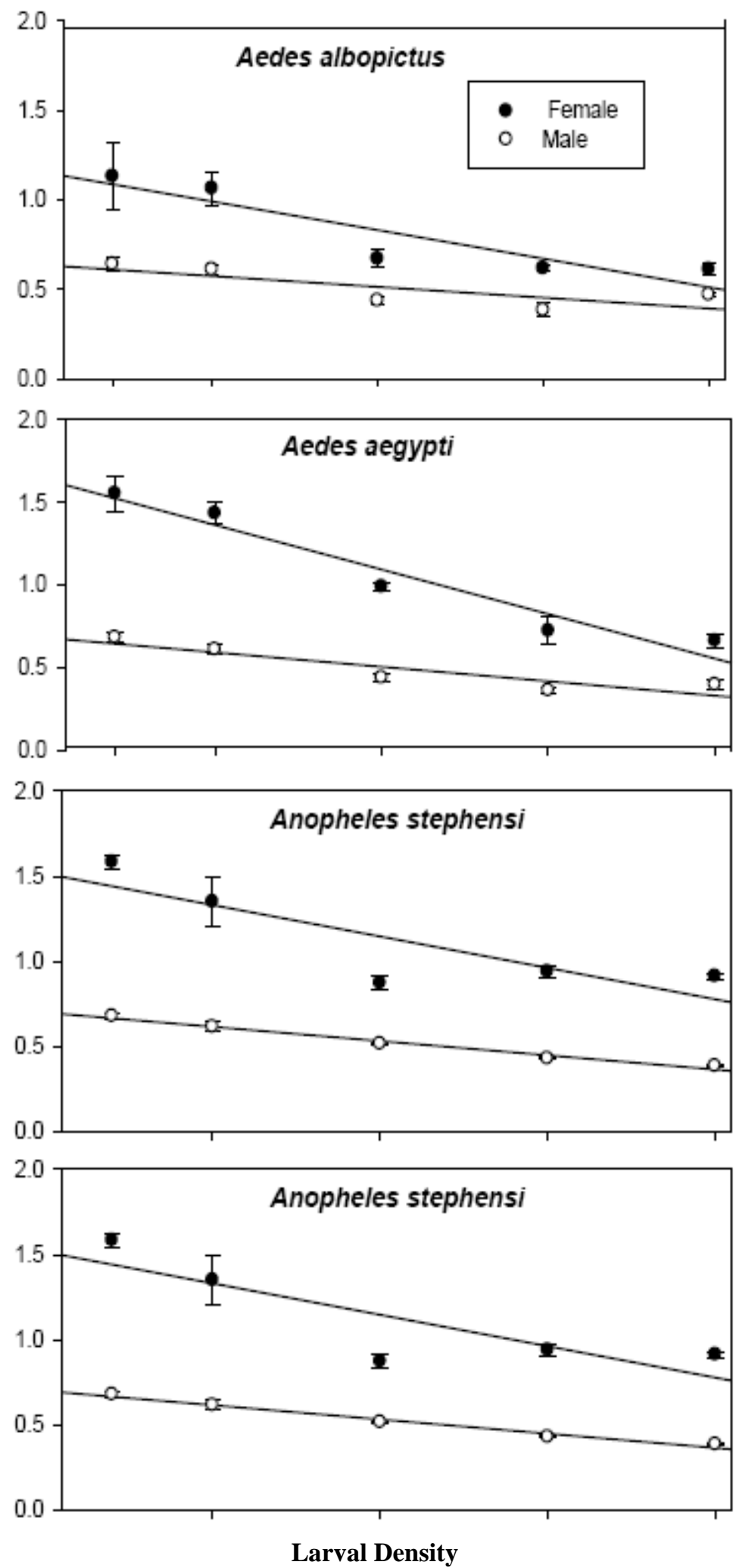

Figure 4. Association between Larval density and adult dry weight among different mosquito species.

In this study maximum survival was observed in Aedes species as compared to Cx. quinquefasciatus and An. stephensi. The adaptation by different mosquito species to survive in containers as breeding habitats might be the reason of this trend. In general $C x$. quinquefasciatus and An. stephensi prefers 
Overcrowding Effects on Larval Development of Four Mosquito species Aedes albopictus, Aedes aegypti, Culex quinquefasciatus and Anopheles stephensi

permanent large water bodies as breeding habitats while Aedes may breed in small temporary water containers $(21,22)$. Consequently Aedes species have to be more adaptable against the stress, produced during competition for resources. Therefore Aedes showed less susceptibility to the competitive environment as compared to An. stephensi and $C x$. quinquefasciatus species. Moreover this phenomenon is complicated and may be species and condition specific. Resource competition in temporary and small water containers could undergo intra-specific or inter-specific competition in mosquito immature and leads to greater variation in adult fitness than species that breed in permanent habitats ${ }^{(23,24)}$.

All the four species showed deviation from 1:1 sex ratio in higher densities. Survival of maximum number of males and less number of females in higher larval densities could be the result of intraspecific competition. These observations are consistent with the previous reports of the study of larval competition ${ }^{(13,25)}$. In general female mosquito requires more nutrition to bring forward their generation and for longer duration of lifecycle, as compared to male. This might be the reason that female died in paucity of sufficient nutrition and male can survive in such a low amount of food in the condition of overcrowding. As female's reproductive success improves more than male's by being larger, their minimal and optimal larval developmental time is expected to be longer and likely more sensitive to deterioration of growth conditions ${ }^{(26,27)}$.

Positive relationship between development time and larval density was reported in the present study for all four species. At higher larval densities growth retarding factors produced by individuals, may function as growth reducing agents or prolonged larval development ${ }^{(28)}$. Clements AN (1992) ${ }^{(11)}$ suggested that larvae of container-breeding mosquito species, subjected to long periods of food shortage, are able to substantially extend their development period.

An. stephensi and $C x$. quinquefasciatus larvae represented less survival rate and shorter development time as compared to both the Aedes species. In case of $C x$. quinquefasciatus, prominent larval mortality in early instars at medium to higher densities while in An. stephensi cannibalism may be the possible reason for reduction in survivors. In a study for An. stephensi species early instar mortality was attributed to cannibalism ${ }^{(29)}$. Carrieri et al. (2003) ${ }^{(30)}$ found that $C x$. pipiens survivorship was relatively low at higher density levels as compared with Ae. albopictus. Ae. albopictus may be more efficient at resource acquisition than other container-dwelling mosquitoes ${ }^{(31,32)}$. In Aedes low mortality rate was observed (Figure $1 \mathrm{~A} \& \mathrm{~B}$ ) which leads to strong competition among larvae for resources as compared to Cx. quinquefasciatus and An. stephensi, where larvae faced less competition due to large mortality at early instars. Consequently Aedes larvae survive for long periods to complete their metamorphosis influenced with growth retarding factors whereas in $C x$. quinquefasciatus and An. stephensi, in absence of such factors remaining live larvae reached to pupation earlier.

Larval crowding in the habitat caused longer duration of immature stage ${ }^{(33)}$ and this hinders the fitness of adult with reduction in weight ${ }^{(34,35)}$. This study explored that the degree of intra-specific competition had a significant effect on adult fitness. In terms of adult dry weight, all the four species followed the same trend of producing light weighed adult from high densities. In the results it has been seen that the body weight of adults from 150 and 200 larvae / container cohorts was slightly more than the adults from lower larval density. Walsh et al. (2012) ${ }^{(36)}$ hypothesized that for this discrepancy, there is a tradeoff between the rate of larval development and body size. Larvae that develop slower may have an opportunity to grow larger because as more time passes, the container is accumulating more food and over the time there is large larval mortality in early stages, very few larvae survives from competition.

High levels of intra- or inter-specific larval competition hamper the adult physical development which leads to the production of susceptible mosquitoes for disease transmission. Disease transmission requires the establishment of virus in the mosquito midgut. For this process numerous morphological (e.g. basement membranes) and physiological (e.g. virus modulating factors) barriers within the mosquito has to overcome by the virus ${ }^{(37,38)}$ and ultimately the dissemination of disease. Pathogen barriers become feeble in physically underdeveloped adults and thus make more susceptible for viral infections. Grimstad and Walker (1991) ${ }^{(39)}$ observed differences in midgut morphology of different sized Aedes triseriatus mosquitoes, raised in different larval nutritions, and subsequent effect of on vector competence. These factors of population dynamics are important for planning any vector control strategies. 
Overcrowding Effects on Larval Development of Four Mosquito species Aedes albopictus, Aedes aegypti, Culex quinquefasciatus and Anopheles stephensi

Thus the study is important in establishing the relationship between models of mosquito population regulation and epidemiological models of disease transmission. In the present study we have established that under laboratory conditions, density-dependent competition and alterations in the availability of nutrients influence the mosquito performance; and physiology in ways that have consequences for life expectancy. If these results interpret to populations in nature, environmental conditions that promote nutrient deprivation and density-dependent competition may influence adult performance, including longevity, and the associated risk for pathogen transmission. Models of mosquito-borne disease transmission are sensitive to changes in adult longevity parameters, which suggest that even small changes in adult longevity caused by larval competition may result in large changes in disease ${ }^{(40,41)}$.

\section{CONCLUSION}

Vector control strategies can also be planed according to the population dynamics of particular region. As the susceptibility of mosquito alters with the population carrying capacity of larval habitat, justified means of vector control should be used. This information will also help to standardize rearing of physically mosquito colonies in laboratory conditions for various experiments.

\section{ACKNOWLEDGEMENT}

The authors are thankful to Dr. Lokendra Singh, Director, DRDE, Gwalior, Madhya Pradesh, India for providing all necessary facility to conduct this research work. Sincere thanks also to the scientists and supportive staff of Vector Management Division for their kind cooperation for carrying out the above work.

\section{REFERENCES}

[1] McIntyre, N. Ecology of urban arthropods: a review and a call to action. Annals of the Entomol. Soc. Am. 93, 825-835 (2000).

[2] Amusan, A.A.S., Mafiana, C.F., Idowu, A.B., Olatunde, GO. Sampling mosquitoes with CDC light traps in rice field and plantation communities in Ogun State, Nigeria. Tanzania Health Res. Bull. 7, 111-116 (2005).

[3] Aigbodion, F.I., Uyi, O.O. Temporal Distribution of and habitat diversification by some mosquitoes (Diptera: Culicidae) species in Benin City. Nigeria J. Entomol. 10(1), 13-23 (2013).

[4] Mafiana, C.F., Annaeema, L.O., Olatunde, G.O. Breeding Sites of larval mosquitoes in Abeokuta, Nigeria. Nig. J. Entomol. 15, 136-143 (1998).

[5] Aigbodion, F.I., Odiachi, F.C. Breeding sites preferences of Anopheline Mosquitoes in Benin City Nigeria. Nigeria J. Entomol. 20, 1-7 (2003).

[6] Lounibos, L.P. Invasions by insect vectors of human disease. Ann. Rev. Entomol. 47, 233- 266 (2002).

[7] Juliano, S.A., Lounibos, L.P., O’Meara, G.F. A field test for competitive effects of Aedes albopictus on Aedes aegypti in South Florida: differences between sites of coexistence and exclusion? Oecologia. 139, 583-593 (2004).

[8] Gimnig, J.E., Ombok, M., Otieno, S., Kaufman, M.G., Vulule, J.M., Walker, E.D. Density-dependent development of An. gambiae (Diptera: Culicidae) larvae in artificial habitats. J. Med. Entomol. 39, 162172 (2002).

[9] Anderson, R.M., May, R.M. Infectious diseases of humans: dynamics and control. Oxford, UK: Oxford University Press (1991).

[10] Rogers, D.J., Randolph, S.W., Snow. R.W., Hay, S.I. Satellite imagery in the study and forecast of malaria. Nature. 415, 710-715 (2002).

[11] Clements, A.N. The biology of mosquitoes: development, nutrition and reproduction. London: Chapman \& Hall (1992).

[12] Dwyer, G., Dushoff, J., Elkinton. J.S., Levin, S.A. Pathogen-driven outbreaks in forest defoliators revisted: building models from experimental data. The American Naturalist 156: 105-120 (2000).

[13] Suleman, M. The effects of intraspecific competition for food and space on the larval development of $C x$. quinquefasciatus. Mosq. News. 42, 347-356 (1982).

[14] Sokal. R.R., Rohlf, F.J. Biometry. The Principles and Practice of Statistics in Biological Research (2nd edition). W. H. Freeman and Co., San Francisco: 859 pp (1981).

[15] Kuno, G., Moore, C.G. Production of larval growth retardant in axenic cultures of Aedes aegypti. Mosq. News 35, 199-201 (1975). 
Overcrowding Effects on Larval Development of Four Mosquito species Aedes albopictus, Aedes aegypti, Culex quinquefasciatus and Anopheles stephensi

[16] Dye, C. Intraspecific competition amongst larval Aedes aegypti: food exploitation or chemical interference? Eco. Entomol. 7 (1), 39-46 (1982).

[17] Bédhomme, S., Agnew, P., Sidobre, C., Michalakis, Y. Pollution by conspecifics as a component of intraspecific competition among Aedes aegypti larvae. Eco. Entomol. 30, 1-7 (2005).

[18] Renshaw, W., Service, M.M., Birley, M.H. Density-dependent regulation of Aedes cantans (Diptera: Culicidae) in natural and artificial populations. Ecol. Entomol. 18, 223-233 (1993).

[19] Gleiser, R.M., Urrutia, J., Gorla, D.E. Effects of crowding on populations of Aedes albifasciatus larvae under laboratory conditions. Entomol Experi. et. Appli. 95, 135- 140 (2000).

[20] Asahina, Syoziro. Food material and feeding procedures for mosquito larvae. Bull. Org. Mond. Sante. Bulletin of World Health Organization. 31, 465-466 (1964).

[21] Vezzani, D. Review: Artificial container-breeding mosquitoes and cemeteries: a perfect match. Trop. Med. Int. Health 12 (2), 299-313 (2007).

[22] Adeleke, M.A., Mafiana, C.F., Idowu, A.B., Adekunle, M.F., Sam-Wobo, S.O. Mosquito larval habitats and public health implications in Abeokuta, Ogun State Nigeria. Tanz. J. of Health Res. 10, 103-107 (2008).

[23] Haramis, L.D. Larval nutrition, adult body size, and the biology of Aedes triseriatus. Ecology of Mosquitoes: Proceedings of a Workshop (ed. by L. P. Lounibos, J. R. Rey \& J. H. Frank), pp. 431-437. Florida Med Entomol Labo Vero Beach, FL (1985).

[24] Nasci, R.S. Adult body size and parity in field populations of the mosquitoes, An. stephensi crucians, Aedes taeniorhynchus and Aedes sollicitans. J. Am. Mosq. Control Assoc. 3, 636-637 (1987).

[25] Reiskind, M.H., Wilson, M.L. Interspecific competition between larval Cx. quinquefasciatus restuans Theobald and $C x$. quinquefasciatus pipiens L. (Diptera: Culicidae) in Michigan. J. Med. Entomol. 45, 2027 (2008).

[26] Agnew, P., Haussy, C., Michalakis, Y. Effects of density and larval competition on selected life history traits of Cx. quinquefasciatus pipiens quinquefasciatus (Diptera: Culicidae). J. Med. Entomol. 37, 732735 (2000).

[27] Bedhomme, S., Agnew, P., Sidobre, C., Michalakis, Y. Sex-specific reaction norms to intraspecific larval competition in the mosquito Aedes aegypti. J. Evol. Bio. 16, 721-730 (2003).

[28] Ikeshoji, T., Mulla, M.S. Overcrowding factors of mosquito larvae. 2. Growth retarding and bacteriostatic effects of the overcrowding factors of mosquito larvae. J. Eco. Entomol. 63, 1737-1743 (1970).

[29] Roy, D.N. On the breeding habits of An. stephensi Liston as observed in laboratory. Ind. J. Med. Res. 19, 635-639 (1931).

[30] Carrieri. M,, Bacchi. M,, Bellini. R,, Maini, S. On the competition occurring between Aedes albopictus and Cx. quinquefasciatus pipiens (Diptera: Culicidae) in Italy. Environ Entomol. 32, 1313-1321 (2003).

[31] Daugherty, J.P., Alto, B.W., Juliano, S.A. Invertebrate carcasses as a resource for competing Aedes albopictus and Aedes aegypti (Diptera: Culicidae). J. Med. Entomol. 37, 364-372 (2000).

[32] Yee, D.A,, Kesavaraju. B., Juliano, S.A. Larval feeding behavior of three co-occurring species of container mosquitoes. J. Vect. Ecol. 29, 315-322 (2004).

[33] Reisen, W.K., Milby, M.M., Bock, M.E. The Effects of Immature stress on selected events in the lifehistory of Cx. quinquefasciatus tarsalis. Mosq. News. 44, 385-395 (1984).

[34] Bradshaw, W.E., Holzapfel, C.M. Reproductive consequences of density-dependent size variation in the pitcher plant mosquito, Wyeomyia smithii (Diptera: Culicidae). Ann. Entomol. Soc. of Am. 85, 274-281 (1992).

[35] Hawley, W.A. The effect of larval density on adult longevity of a mosquito, Aedes sierrensis: epidemiological consequences. J. Anim. Eco. 54, 955-964 (1985).

[36] Walsh, R.K., Bradley, C., Apperson, C.S., Gould, F. An Experimental Field Study of Delayed Density Dependence in Natural Populations of Aedes albopictus. Plos One. 7(4), e35959. doi:10.1371/journal.pone.0035959 (2012).

[37] Woodring, J.L., Higgs, S., Beaty, B.J. Natural cycles of vector-borne pathogens. In The biology of disease vectors (eds B. J. Beaty \& W. C. Marquardt), pp. 51-72, Niwot, CO: University Press of Colorado (1996).

[38] Ader, D.B. Modulation of dengue virus infection of dendritic cells by Aedes aegypti saliva. Viral Immuno. $17,252-265$ (2004).

[39] Grimstad, P.R., Walker, E.D. Aedes triseriatus (Diptera: Culicidae) and LaCrosse virus. IV. Nutritional deprivation of larvae affects the adult barriers to infection and transmission. J. Med. Entomol. 28, 378-386 (1991). 
Overcrowding Effects on Larval Development of Four Mosquito species Aedes albopictus, Aedes aegypti, Culex quinquefasciatus and Anopheles stephensi

[40] Luz, P.M., Codec,o, C.T., Massad, E., Struchiner, C.J. Uncertainties regarding dengue modeling in Rio de Janeiro. Memórias do Instituto Oswaldo Cruz. 98, 871-878 (2003).

[41] Alto, B.W., Lounibos, L.P., Mores, C.N., Reiskind, M.H. Larval competition alters susceptibility of adult Aedes mosquitoes to dengue infection. Proceeding Royal Society. 275, 463-471 (2008).

Citation: R. Yadav et al., " Overcrowding Effects on Larval Development of Four Mosquito species Aedes albopictus, Aedes aegypti, Culex quinquefasciatus and Anopheles stephensi ", International Journal of Research Studies in Zoology, vol. 3, no. 3, p. 1-10, 2017. http://dx.doi.org/10.20431/2454-941X.0303001

Copyright: (c) 2017 Authors. This is an open-access article distributed under the terms of the Creative Commons Attribution License, which permits unrestricted use, distribution, and reproduction in any medium, provided the original author and source are credited. 